\title{
Portfolio Risk and Dependence Modeling
}

\author{
Arsalan Azamighaimasi \\ School of Management, Wuhan University of Technology \\ 205 Luoshi Road, Wuhan 430070, Hubei, China \\ Tel: 86-134-7607-5493Ｅ-mail: Arsalan.azami2011@gmail.com
}

Received: July 18, 2012

doi:10.5430/ijfr.v4n1p151
Accepted: December 10, $2012 \quad$ Online Published: December 23, 2012

URL: http://dx.doi.org/10.5430/ijfr.v4n1p151

\begin{abstract}
This paper has considered portfolio credit risk with a focus on two approaches, the factor model, and copula model. We have reviewed two models with emphasis on the joint default probably. The copula function describes the dependence structure of a multivariate random variable, in this paper, it used as a practical to simulation of generate portfolio with different copula, and we only used Gaussian and t-copula case. We generated portfolio default distributions and studied the sensitivity of commonly used risk measures with respect to the approach in modeling the dependence structure of the portfolio.
\end{abstract}

Keywords: Gaussian copula, Factor model, Copula model

\section{Introduction}

We need to know components of portfolio risk and their interaction. The Basel Committee for Banking Supervision in its Basel II proposals to develop an appropriate framework for a global financial regulation system; seeBIS (2001). Several portfolio credit risk models developed in the industry have been made public; e.g., CreditMetrics (Gupton et al. 1997), CreditRisk+ (Credit Suisse Financial Products 1997) and Credit Portfolio View (Wilson 1997a and 1997b). Others remain proprietary, such as KMV's Portfolio Manager (Kealhofer 1996). Although the models seem quite different on the surface, recent theoretical work has shown an underlying mathematical equivalence among them (Gordy 2000; Koyluoglu and Hickman 1998). The credit portfolio models to achieve portfolio loss distributions, which are models, can be classified as based on credit rating systems; See Crouhyet al. (2001) foran exact description and analysis of the various models. Frey and McNeil (2001) studied of mathematical side of the models, and they considered the modeling of dependent defaults in large credit portfolios using la-tent variable models and mixture models. Crouhy et al. (2000) compared and reviewed models on the benchmark portfolio like; the credit migration approach, the structural approach, the actuarial approach, and McKinsey approach.However, few studies have attempted to investigate aspects of portfolio risk based on rating-based credit risk models thoroughly. Gordy (2000)offered comparative anatomy of two particularly influential benchmarks for credit risk models, the Risk Metrics Group's Credit Metrics and Credit Suisse Financial Product’sCreditRisk ${ }^{+}$‡Kiesel et al. (1999) employ a "mark-to-market" model and stress the importance of stochastic changes in credit spreads associated with market values - an aspect also highlighted in Hirtle et al. (2001).

The aim of this paper is to contribute to the understanding of the performance of rating-based credit portfolio models. We applied a default-mode model to assess the impact of changing dependence structure within the portfolio. First, we discussed the copula model as one of the dependency approaches within the portfolio. Second, we mentioned about a factor model and focus on the effects of default dependence model on within the portfolio.Eventually, we simulated types of copula model with different degree of freedom within the portfolio.

\section{Copula Modeling}

This part we want an overview of basic copula used in structural and models. Copulas provide a natural way to study and measure dependence between random variables. Suppose we have specified a portfolio of $n$ obligors, with default times $\tau_{1}, \tau_{2}, \ldots \tau_{n}$. The variable of default of obligori, $i=1,2, \ldots n$, at time $t$, is donated as $Y_{i}(t):=1_{\left\{\tau_{i \leq t}\right\}}$. The probability space is $(\Omega, \mathcal{H}$.P $)$. This space has filtration $\mathbb{G}_{i}:=\left\{\mathcal{G}_{i t} ; t \geq 0\right\}$ :

$$
\mathcal{G}_{i t}:=\sigma\left(Y_{i}(u) ; 0 \leq u \leq t\right)
$$


For the joint default probability at time $t$, evaluated at time 0 , as $F(t)$

$$
F(t):=P\left(Y_{1}(t)=1, Y_{2}(t)=1, \ldots Y_{n}(t)=1 \mid \mathcal{G}_{0}\right)
$$

The survival property as $s(t)$

$$
s(t):=P\left(Y_{1}(t)=0, Y_{2}(t)=0, \ldots Y_{n}(t)=0 \mid \mathcal{G}_{0}\right)
$$

We take for granted the copula definition as a joint distribution function with uniform margins, which implies that $C$ and take for granted the fundamental Sklar's theorem, in terms of a copula $C$ and the marginal distribution functions $F_{i}(t), i=1,2, \ldots, n$ :

$$
F(t)=C\left(F_{1}(t), F_{2}(t), \ldots, F_{n}(t)\right)
$$

The joint survival probability $s(t)$ with survival copula, $\breve{C}$ and the marginal survival functions $S_{i}(t):=1-F_{i}(t)$ :

$$
S(t)=\check{C}\left(S_{1}(t), S_{2}(t), \ldots S_{n}(t)\right)
$$

Factor copula $C^{\perp}$ is,

$$
C^{\perp}\left(u_{1}, \ldots, u_{n}\right)=u_{1} \times u_{2} \times \ldots \times u_{n} .
$$

In the credit risk case, since the variables $\tau_{i}$ are default time, the copula represents default dependence. It is donated as $C^{\tau}$,

$$
\begin{aligned}
F(t) & =C^{\tau}\left(F_{1}(t), F_{2}(t), \ldots, F_{n}(t)\right) \\
S(t) & =\check{C}^{\tau}\left(S_{1}(t), S_{2}(t), \ldots S_{n}(t)\right)
\end{aligned}
$$

According to Merton model (1974) if default of firm (i) occurs, the values of asset or values of shares cross from barrier line of outstanding debt at debt maturity. Default occurred when the firm's asset value $V_{i}(t)$ falls to the liability one, $K_{i}(t)$.the time of default is;

$$
\tau_{i}= \begin{cases}t & P\left(V_{i}(t) \leq K_{i}(t)\right) \\ +\infty & P\left(V_{i}(t)>K_{i}(t)\right)\end{cases}
$$

The default probability at time $t$ is

$$
F_{i}(t)=P\left(V_{i}(t) \leq K_{i}(t)\right)
$$

The marginal default probability can be easily computed to be

$$
F_{i}(t)=\Phi\left(d_{2 i}(t)\right)
$$

Then,

$$
d_{2 i}(t):=\frac{\ln \left(\frac{V_{i}(0)}{K_{i}(t)}\right)+\left(m-\frac{\sigma^{2}}{2}\right) t}{\sigma \sqrt{t}}
$$

And $m$ is the instantaneous return on assets, which equates the riskless rate $r$ under the risk neutral measure. The joint default probability of $n$ assets is

$$
\left.F_{1}(t)=P\left(V_{1}(t) \leq K_{1}(t)\right), \ldots V_{n}(t) \leq K_{n}(t) ; \mathcal{G}_{0}\right)=\Phi_{R}\left(-d_{21}(t), \ldots,-d_{2 n}(t)\right)
$$

Where $\Phi_{R}$ is the distribution function of a standard normal vector with correlation matrix R. the marginal default probabilities is follows

$$
F(t)=\Phi_{R}\left(\Phi^{-1}\left(F_{1}(t)\right), \ldots, \Phi^{-1}\left(F_{n}(t)\right)\right)
$$

To study the effect of different copula on default correlation, we use the following examples of copula (further details on these copula can be found in Embrechts et al. (2001)).

Gaussian copula:

$$
C_{R}\left(u_{1}, \ldots, u_{n}\right)=\Phi_{R}^{n}\left(\Phi^{-1}\left(u_{1}\right), \ldots, \Phi^{-1}\left(u_{n}\right)\right)
$$

Where $\Phi_{R}^{n}$ denotes the joint distribution function of the $n$-variety normal with linear correlation matrix $R$, and $\Phi^{-1}$ the inverse of the distribution function of the univariate standard normal. 
A student $t$ copula:

$$
c_{v, R}\left(u_{1}, \ldots, u_{n}\right)=t_{v, R}^{n}\left(t_{v}^{-1}\left(u_{1}\right), \ldots, t_{v}^{-1}\left(u_{n}\right)\right)
$$

Where $t_{v, R}$ is the standardized multivariate Student's $t$ distribution, withcorrelation matrix $R$ and $v$ degrees of freedom, While $t_{v}^{-1}$ is the inverse of the corresponding margin.

Gumbel copula:

$$
C_{\theta}\left(u_{1}, \ldots, u_{n}\right)=\exp \left\{-\left[\left(-\log u_{1}\right)^{\theta}+\cdots+\left(-\log u_{n}\right)^{\theta}\right]^{1 / \theta}\right\}
$$

Where $\theta \in[1, \infty)$. This class of copula is a sub-class of the class of Archimedean copula. According to the Table 1, joint default probabilities of two obligors represented through three types of obligors with individual default probabilities corresponding to rating classes.as you will see that tand Gumbel copula have higher joint default probabilities than the Gaussian copula.

\section{Factor Modeling}

The other way to default modeling allows us to switch to the so called product copula. The reduction technique, which widely adopted for the assessment of losses in high-dimensional portfolioswith hundreds of obligors (see for instance Laurent and Gregory (2003)), is the standard approach of (linear) factorization, or transformation into a Bernoulli factor model.

In the typical portfolio analysis, the vector $V$ embedded in a factor model, which allows for easy analysis of correlation, the typical measure of dependence. We assume that the underlying variables $V_{j}$ are driven by a vector of common factors.

$$
V_{j}=\sum_{i=1}^{p} a_{i j} Z_{i}+\sigma_{j} \epsilon_{j}, j=1, \ldots, n \quad Z \sim N\left(0, \sum\right)
$$

Where $p$ is dimensional normal vector, and $\in$ is independent normally distributed random variables. Here $a_{i j}$ is obligor $j$ to factori, i.e. the so-called factor loading and $\sigma_{j}$ is volatility of the risk contribution. The default indicators $Y_{j}(t)$ of the $n$ obligor are independent Bernoulli variables, with probability:

$$
Y_{j}(t)=\left\{\begin{array}{cc}
1 & V_{j} \leq K_{j} \\
0 & \text { otherwise }
\end{array}\right.
$$

Where $K_{j}$ is cut-off point for default obligor $j$. The individual default probabilities are

$$
F_{j}=P\left(Y_{j}=1\right)=P\left(V_{j} \leq K_{j}\right) \text {, }
$$

And the joint default probability is

$$
F_{i j}=P\left(Y_{i}=1, Y_{j}=1\right)=P\left(V_{i} \leq K_{i}, V_{j} \leq K_{j}\right) .
$$

If we denote by $\rho_{i j}=\operatorname{Corr}\left(V_{i}, V_{j}\right)$ the correlation of the underlying latent variables and by $\rho_{i j}^{K}=\operatorname{Corr}\left(Y_{i}, Y_{j}\right)$ the default correlation of obligors $i$ and $j$, then we obtain the default correlation formula

$$
\rho_{i j}^{K}=\frac{F_{i j}-F_{i} F_{j}}{\sqrt{F_{i} F_{j}\left(1-F_{i}\right)\left(1-F_{j}\right)}}
$$

Under assumption above, we obtain the joint default probability

$$
F_{i j}=\int_{-\infty}^{K_{i}} \int_{-\infty}^{K_{j}} \varphi\left(u, v ; \rho_{i j}\right) d u d v
$$

Where $\varphi(u, v ; \rho)$ is bivariate normal density with correlation coefficient.

\section{Simulation Copula Model}

Here, we want to generate portfolios with given marginal and the above copula.We only use Gauss and $t-$ copula case. We are looking for a random sample generation for this mean we obtain the generation of an $n$-variety normal 
with liner correlation matrix $R,\left(x_{1}, \ldots, x_{n}\right)^{\top} \sim N(0, R)$,to take realizations from a Gaussian copula we simply have to transform the marginal:

- $\quad$ Set $u_{i}=\Phi\left(x_{i}\right), \quad i=1, \ldots, n$

- $\left(u_{1}, \ldots, u_{n}\right)^{\top} \sim C_{R}^{\text {Gauss }}$

To generate random varieties from the $t$-copula $C_{v, R}^{t}$ we assume the random vector $\mathrm{X}$ act the stochastic process

$$
X=\mu+\sqrt{\frac{v}{Z}} Y \text { (in distribution) }
$$

With

$$
\mu \in \mathbb{R}^{n}, \quad Z \sim \chi_{v}^{2} \text { and } Y \sim N\left(0, \sum\right)
$$

Where $\mathrm{Z}$ and $\mathrm{Y}$ are independent, and then $\mathrm{X}$ is $t_{v}$ distributed with mean $\mu$ and covariance matrix $\left(\frac{v}{v-2} \sum\right)$. weassume $v>2$, while the stochastic process is still valid the parameters has to change for $v \leq 2$. We will have algorithm (this is algorithm in Embrechts et al. (2001)):

- $\quad$ Set $x=\sqrt{\frac{v}{z}}$

- Set $u_{i}=t_{v}\left(x_{i}\right), \quad i=1, \ldots, n$

- $\left(u_{1}, \ldots, u_{n}\right)^{\top} \sim C_{v, R}^{t}$.

We can replace the $u_{i}$ with $\Phi^{-1}\left(u_{i}\right)$ in order to have multivariate distribution with $t$-copula and normal marginal, to obtain the $t-\operatorname{copula} C_{v, R}^{t}$.

As see the figure 1 represents tree types of observations from a multivariate normal distribution with mean vector mu and covariance matrix. The figure 2 shows to computes a scatterplot of a normal sample and in a second plot the contour ellipses for $m u=\#(3,2)$ and sigma $=\#(1,-1.5) \sim \#(-1.5,4)$ with different observations.

\section{Portfolio}

For first simulation exercise, we assume that the underlying variables $V_{j}$ are normally distributed within a single factor framework, i.e. $p=1$ and $a_{j 1}$ in the formula as follow:

$$
V_{j}=\sum_{i=1}^{p} a_{i j} Z_{i}+\sigma_{j} \epsilon_{j}, j=1, \ldots, n
$$

They are constant and chosen so that the correlation for the underlying latent variables $V_{j}$ is $\rho=0.2$ (Kiesel et al. (1999).note that We use three rating classes, named A,B,C with default probabilities $0.005,0.05,0.15$ roughly corresponding to default probabilities from standard rating classes, Ong (1999). To generate different degrees of tail correlation, we link the individual assets together using a Gaussian, a $t_{10}$ and a $t_{4}$-copula.

The table's follows represents of the effect tail-dependence has on the high quintiles of highly-rated portfolios, the 99\%-quantile for the $t_{4}$-copula is more than three-times larger than the corresponding quintile for the Gaussian copula.

The same effect can be observed for lower rated portfolios although not quite with a similar magnitude.

As expected results in Tables 5, 6, 7 show increase in the quantiles due to the increased correlation within the portfolio. However, comparing the three tables we will see that the sensitivity of the portfolio loss quantilesis higher with regard to the underlying copula than to the correlation within the portfolio.

\section{Conclusion}

To investigate the riskiness of credit-risky portfolios is one of the big challenging in the finance area. One of an important thing for a model of credit-risky portfolios is the dependence structure of the underlying obligors. We 
studied two approaches, a factor structure, and the direct specification of a copula. We generated portfolio default distributions and studied the sensitivity of commonly used risk measures with respect to the approach in modeling the dependence structure of the portfolio. My simulation results indicate that the degree of tail dependence of the underlying copula plays a crucial role as a credit risk, and the copula linking the underlying variables together is of crucial importance especially for portfolios of highly rated obligors.

\section{References}

BIS. (2001). Overview of the new Basel capital accord. Basel Committee on Banking Supervision.

Caouette, J., Altman, E., \& Narayanan, P. (1998). Managing Credit Risk, The Next Great Financial Challenge. Wiley Frontiers in Finance, Vol. Wiley Frontiers in Finance, Wiley \& Sons, Inc, New York.

Carey, M. (2000). Dimensions of credit risk and their relationship to economic capital requirements. Federal Reserve Board.

Crouhy, M., Galai, D., \& Mark, R. (2000). A comparative analysis of current credit risk models. ournal of Banking and Finance, 24(1-2), 59-117.

Crouhy, M., Galai, D., \& Mark, R. (2001). Risk management. McGraw Hill.

Embrechts, P., Lindskog, F., \& McNeil, A. (2001). Modelling dependence with copulas and applications to risk management. ETH Zürich.

Frey, R., \& McNeil, A. (2001). Modelling dependent defaults. ETH Zürich.

Gordy, M. (2000). A comparative anatomy of credit risk models. Journal of Banking and Finance, 24, 119-149.

Jorion, P. (2000). Value at Risk. New York. : McGraw-Hill.

Kiesel, R., Perraudin, W., \& Taylor, A. (1999). The structure of credit risk. Birkbeck College.

Koyluoglu, H., \& Hickmann, A. (1998). A generalized framework for credit portfolio models. Wyman \& Company.

Luciano, E. (2008). Copula-based default dependence modelling: where do we stand?

Morgan, J. (1997). Creditmetrics. New York: JP Morgan.

Nickell, P., Perraudin, W., \& Varotto, S. (1998). Ratings-versus equity-based credit risk models: An empirical investigation. Unpublished Bank of England mimeo.

Ong, M. (1999). Internal Credit Risk Models. Capital Allocation and Performance Measurement. London: Risk Books.

Table 1. Copula and default probability

\begin{tabular}{|c|c|c|c|}
\hline \multicolumn{1}{|c|}{ copula } & \multicolumn{2}{|c|}{ Default probability } \\
\hline & Class $\mathrm{A}\left(\times 10^{-6}\right)$ & Class $\mathrm{B}\left(\times 10^{-4}\right)$ & 52.45 \\
\hline$*[-2 p t]$ Gaussian & 6.89 & 3.38 & 71.03 \\
\hline$C_{10}^{t}$ & 46.55 & 7.88 & 97.96 \\
\hline$C_{4}^{t}$ & 134.80 & 15.35 & 144.56 \\
\hline Gumbel, $\quad C_{2}$ & 57.20 & 14.84 & 283.67 \\
\hline Gumbel, $\quad C_{4}$ & 270.60 & 41.84 & $\left.10^{-4}\right)$ \\
\hline
\end{tabular}


Table 2. Effect of normal copula with default probability 0.005

\begin{tabular}{|ll|l|l|l|l|}
\hline Portfolio & Copula & Mean & variance & $\boldsymbol{a}=\mathbf{0 . 9 5}$ & $\boldsymbol{a}=\mathbf{0 . 9 9}$ \\
$\mathrm{A}=1000$ & normal & 0.115 & 0.13391 & 1 & 2 \\
$\mathrm{~A}=500$ & normal & 0.106 & 0.119 & 1 & 1 \\
$\mathrm{~A}=50$ & normal & 0.18 & 0.19143 & 1 & 2 \\
$\mathrm{~B}=1000$ & normal & 0.99 & 1.8277 & 4 & 6 \\
$\mathrm{~B}=500$ & normal & 1.038 & 1.8442 & 4 & 6 \\
$\mathrm{~B}=50$ & normal & 1.18 & 2.3955 & 4 & 6 \\
$\mathrm{C}=1000$ & normal & 3.029 & 7.0953 & 8 & 11 \\
$\mathrm{C}=500$ & normal & 2.998 & 6.9078 & 8 & 11 \\
$\mathrm{C}=50$ & normal & 3.1 & 7.3163 & 9 & 10 \\
\hline
\end{tabular}

Table 3. Effect of $t_{10}-$ copula with default probability 0.05

\begin{tabular}{|l|c|l|l|l|l|} 
Portfolio & Copula & Mean & variance & $\boldsymbol{a}=\mathbf{0 . 9 5}$ & $\boldsymbol{a}=\mathbf{0 . 9 9}$ \\
$\mathrm{A}=1000$ & $t_{10}$ & 0.101 & 0.26907 & 1 & 2 \\
$\mathrm{~A}=500$ & $t_{10}$ & 0.098 & 0.15671 & 1 & 2 \\
$\mathrm{~A}=50$ & $t_{10}$ & 0.14 & 0.36776 & 1 & 4 \\
$\mathrm{~B}=1000$ & $t_{10}$ & 0.963 & 2.38 & 4 & 6 \\
$\mathrm{~B}=500$ & $t_{10}$ & 0.994 & 2.1984 & 4 & 6 \\
$\mathrm{~B}=50$ & $t_{10}$ & 1.06 & 2.9147 & 4 & 9 \\
$\mathrm{C}=1000$ & $t_{10}$ & 3.008 & 7.9799 & 9 & 11 \\
$\mathrm{C}=500$ & $t_{10}$ & 3.05 & 7.9474 & 9 & 12 \\
$\mathrm{C}=50$ & $t_{10}$ & 3.42 & 8.9016 & 9 & 11 \\
\hline
\end{tabular}

Table 4. Effect oft $t_{4}-$ copula with default probability 0.15

\begin{tabular}{|c|c|c|c|c|c|}
\hline Portfolio & Copula & Mean & variance & $a=0.95$ & $a=0.99$ \\
\hline$A=1000$ & $t_{4}$ & 0.088 & 0.39665 & 0 & 2 \\
\hline$A=500$ & $t_{4}$ & 0.084 & 0.24543 & 0 & 2 \\
\hline $\mathrm{A}=50$ & $t_{4}$ & 0.22 & 2.42 & 0 & 11 \\
\hline$B=1000$ & $t_{4}$ & 0.924 & 3.1454 & 5 & 9 \\
\hline$B=500$ & $t_{4}$ & 1 & 3.0261 & 7 & 5 \\
\hline$B=50$ & $t_{4}$ & 1.02 & 3.5302 & 4 & 11 \\
\hline $\mathrm{C}=1000$ & $t_{4}$ & 2.997 & 9.5860 & 10 & 12 \\
\hline $\mathrm{C}=500$ & $t_{4}$ & 3.028 & 9.0213 & 9 & 13 \\
\hline $\mathrm{C}=50$ & $t_{4}$ & 3.34 & 9.2086 & 9 & 12 \\
\hline
\end{tabular}

We assume the second factor, i.e. $p=2$ in (4), for a sub-portfolio of 100 obligors increasing the correlation of the latent variables $V_{j}$ within the sub-portfolio to 0.5 for this reasoning we want to shows the effects of increased correlation within parts of the portfolio; we change the factor loading within parts of the portfolio. 
Table 5. The effect of correlation cluster with default probability 0.005

\begin{tabular}{|l|l|llll|ll|}
\hline portfolio & copula & $\begin{array}{l}\text { First } \\
\text { subportfolio }\end{array}$ & $\begin{array}{l}\text { Second } \\
\text { subportfolio }\end{array}$ & mean & variance & $\mathbf{0 . 9 5}$ & $\mathbf{0 . 9 9}$ \\
\hline $\mathrm{A}=1000$ & normal & 100 & 150 & 1.237 & 6.8447 & 5 & 13 \\
$\mathrm{~A}=500$ & normal & 50 & 75 & 0.6 & 1.6433 & 2 & 7 \\
$\mathrm{~A}=50$ & normal & 20 & 30 & 0.24 & 0.47184 & 1 & 4 \\
$\mathrm{~B}=1000$ & normal & 100 & 150 & 12.723 & 204.41 & 41 & 71 \\
$\mathrm{~B}=500$ & normal & 50 & 75 & 6.198 & 47.951 & 20 & 33 \\
$\mathrm{~B}=50$ & normal & 20 & 30 & 2.58 & 7.3506 & 10 & 11 \\
$\mathrm{C}=1000$ & normal & 100 & 150 & 37.972 & 871.43 & 96 & 132 \\
$\mathrm{C}=500$ & normal & 50 & 75 & 18.832 & 200.1 & 49 & 63 \\
$\mathrm{C}=50$ & normal & 20 & 30 & 7.74 & 30.36 & 20 & 23 \\
\hline
\end{tabular}

Table 6 . Tthe effect of correlation cluster with default probability 0.05

\begin{tabular}{lllllllll} 
portfolio & copula & $\begin{array}{l}\text { First } \\
\text { subportfolio }\end{array}$ & $\begin{array}{l}\text { Second } \\
\text { subportfolio }\end{array}$ & mean & variance & $\mathbf{0 . 9 5}$ & $\mathbf{0 . 9 9}$ \\
$\mathrm{A}=1000$ & $t_{10}$ & 100 & 150 & 1.451 & 27.335 & 7 & 28 \\
$\mathrm{~A}=500$ & $t_{10}$ & 50 & 75 & 0.644 & 6.7668 & 3 & 11 \\
$\mathrm{~A}=50$ & $t_{10}$ & 20 & 30 & 0.2 & 0.32653 & 1 & 3 \\
$\mathrm{~B}=1000$ & $t_{10}$ & 100 & 150 & 11.76 & 299.29 & 52 & 83 \\
$\mathrm{~B}=500$ & $t_{10}$ & 50 & 75 & 6.28 & 85.605 & 24 & 44 \\
$\mathrm{~B}=50$ & $t_{10}$ & 20 & 30 & 2.32 & 11.365 & 10 & 17 \\
$\mathrm{C}=1000$ & $t_{10}$ & 100 & 150 & 38.24 & 1104.7 & 105 & 148 \\
$\mathrm{C}=500$ & $t_{10}$ & 50 & 75 & 18.638 & 263.7 & 52 & 75 \\
$\mathrm{C}=50$ & $t_{10}$ & 20 & 30 & 7.5 & 31.235 & 17 & 24 \\
\hline
\end{tabular}

Table 7 . The effect of correlation cluster with default probability 0.15

\begin{tabular}{|lllllllll|}
\hline portfolio & copula & $\begin{array}{l}\text { First } \\
\text { subportfolio }\end{array}$ & $\begin{array}{l}\text { Second } \\
\text { subportfolio }\end{array}$ & mean & variance & $\mathbf{0 . 9 5}$ & $\mathbf{0 . 9 9}$ \\
$\mathrm{A}=1000$ & $t_{4}$ & 100 & 150 & 1.635 & 70.278 & 7 & 42 \\
$\mathrm{~A}=500$ & $t_{4}$ & 50 & 75 & 0.682 & 14.554 & 3 & 21 \\
$\mathrm{~A}=50$ & $t_{4}$ & 20 & 30 & 0.36 & 2.1943 & 1 & 10 \\
$\mathrm{~B}=1000$ & $t_{4}$ & 100 & 150 & 13.385 & 592.25 & 65 & 128 \\
$\mathrm{~B}=500$ & $t_{4}$ & 50 & 75 & 6.266 & 132.82 & 28 & 61 \\
$\mathrm{~B}=50$ & $t_{4}$ & 20 & 30 & 2.26 & 16.074 & 13 & 18 \\
$\mathrm{C}=1000$ & $t_{4}$ & 100 & 150 & 38.465 & 1395 & 117 & 157 \\
$\mathrm{C}=500$ & $t_{4}$ & 50 & 75 & 18.676 & 331.96 & 56 & 80 \\
$\mathrm{C}=50$ & $t_{4}$ & 20 & 30 & 7.56 & 41.109 & 23 & 27 \\
\hline
\end{tabular}



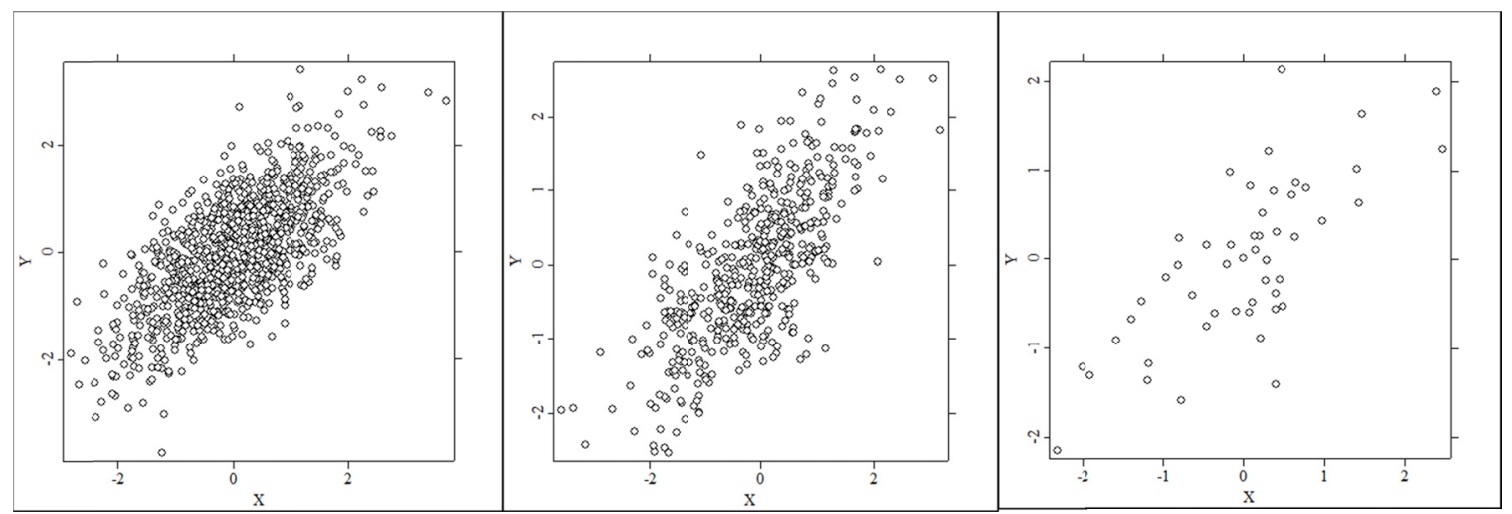

$$
\mathrm{A}=1000 \mathrm{~B}=500 \mathrm{C}=50
$$

Figure 1. Shows $1000,500,50$ observations from a multivariate normal distribution
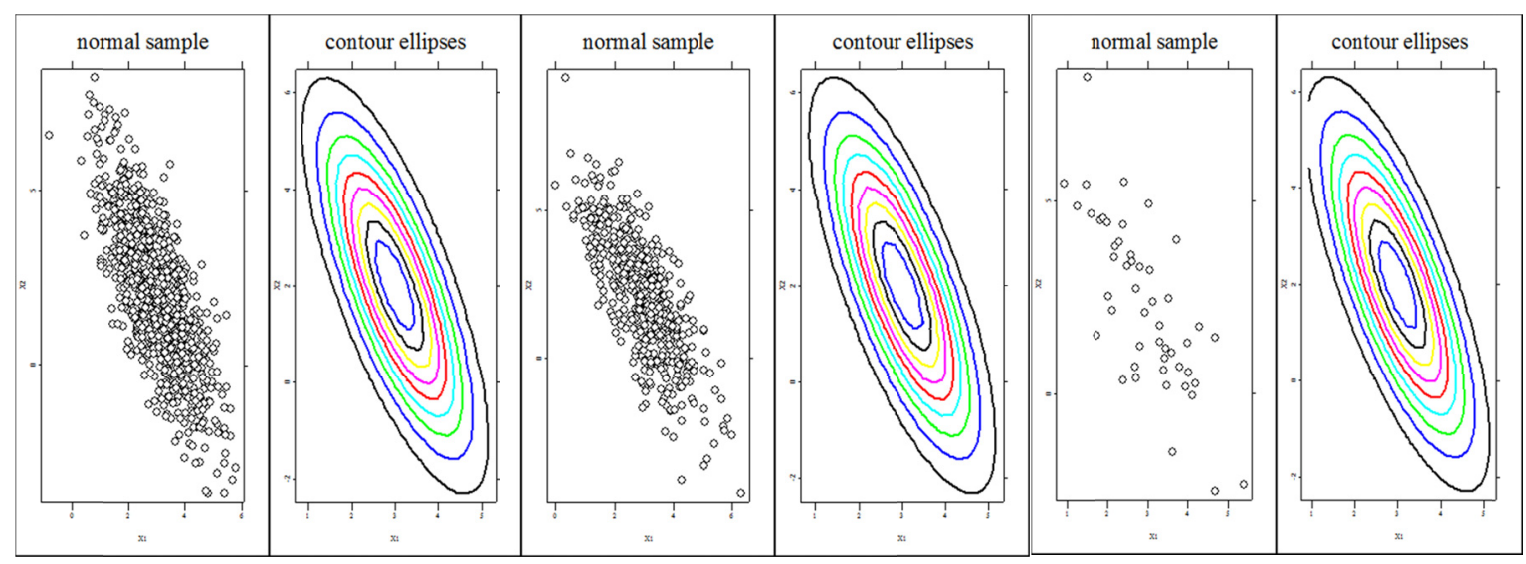

$$
\mathrm{A}=1000
$$

$\mathrm{B}=500$

$\mathrm{C}=50$

Figure 2. Computes a scatterplot of a normal sample and in a second plot the contour ellipses for $m u=\#(3,2)$ and sigma $=\#(1,-1.5) \sim \#(-1.5,4)$ with different size 